\title{
Mutual Engagement in Social Music Making
}

\author{
Nick Bryan-Kinns \\ Interactional Sound and Music Group, Centre for Digital Music \\ Queen Mary University of London, Mile End, London. E1 4NS. UK \\ Tel: +44 (0) 207882 7845; Fax: +44 (0) 2078827064 \\ nick.bryan-kinns@eecs.qmul.ac.uk
}

\begin{abstract}
Mutual engagement occurs when people creatively spark together. In this paper we suggest that mutual engagement is key to creating new forms of multi-user social music systems which will capture the public's heart and imagination. We propose a number of design features which support mutual engagement, and a set of techniques for evaluating mutual engagement by examining inter-person interaction. We suggest how these techniques could be used in empirical studies, and how they might be used to inform artistic practice to design and evaluate new forms of collaborative music making.
\end{abstract}

Keywords. Design, Evaluation, Mutual Engagement, Multi-User, Social Interaction, Human Communication.

\section{Introduction}

Is music dead? Whilst figures in the commercial music industry bemoan the loss of sense of purpose in contemporary music and the role of the Internet in sidelining music as a political force [9], we contend that new technologies hold the key to reinvigorating music's social role. We accept that music has dropped away from being a driving force behind communication technology innovation, losing out to text based networks such as Twitter and Facebook, and argue that what is needed are innovative and engaging ways for people to make, share, enjoy, and experience music within the context of the modern, connected, real-time world we live in. To this end, we are exploring ways to understand the role of audio in multi-person interactions from interactive art, music, and performance through to workplace collaborations. We believe that the key to success in this venture will be designing new multi-person audio experiences which are informed by understandings of human communication, and which exploit the unique opportunities offered by new technologies rather than mimicking existing ways of interacting. In short, music $i s$ dead, long live music!

In this paper we outline our approach to understanding mutual engagement in multi-person music making. We first describe a set of design features which we believe will increase mutual engagement in multi-user systems and social music experiences. We then present a set of techniques for identifying mutual engagement in music making by examining the minutiae of the user interface mediated interaction 
between participants. Finally we present a few illustrative descriptions of multiperson mutually engaging systems we have designed, built, and evaluated. We believe that our approach is suitable for music making and using music to support work.

\section{Mutual Engagement}

Sadly, in interface design, sound has been limited to providing alert cues, or ongoing background awareness in collaborative work situations cf. [1]. Some research has examined audio as a medium for collaborative work [13], and even in the field of New Interfaces for Musical Expression, the evaluation of audio centric interfaces tends to focus on parameter manipulation tasks cf. [19] rather than examining the nature of collaborative audio creativity cf. [2] and how these interfaces could support a creative and engaging experience [8]. To address this, we explore the concept of mutual engagement - points at which people creatively spark together and enter a state of group flow [5] - and examine how different user interfaces features affect people's levels of mutual engagement. In this way we hope to identify and develop more socially engaging musical experiences which will return music to the core of human experience. The key distinguishing characteristic of mutual engagement is: "it involves engagement with both the products of an activity and with the others who are contributing to those products" [ibid]. Points of mutual engagement are indicated by the attunement of participants' actions to each other (e.g. mirroring each others' contributions, or building on each others' compositions), and focused interaction with the product at hand (e.g. careful editing and manipulation of parameters for expressive effect). These points of mutual engagement are essentially points of group flow cf. [7], similar in context to Sawyer's ethnographic descriptions of group flow [16], but we concentrate on analysing the minutiae of the group interaction mediated through the interface in order to inform design of engaging collaborative systems. In order to achieve this, Bryan-Kinns and Hamilton [5] drew on models of human communication e.g. [6] and $\mathrm{CSCW}$ research e.g. [10] to develop a set of design criteria and evaluation measures for mutual engagement which are outlined in the next section.

\subsection{Design Features}

We have identified a number of design features [5][3] which we believe are important to supporting mutually engaging interaction:

- Mutual awareness of action - highlighting new contributions to the joint product, and indicating authorship has been shown to increase mutual engagement.

- Annotation - being able to communicate in and around a shared product, and being able to refer to parts of the product helps participants engage with each other.

- Shared and consistent representations - participants find it easier to understand the state of the joint product, and the effect of their own and others' contributions when the representations are shared and consistent.

- Mutual modifiability - editing each others' contributions increases engagement with each others' product, and the activity becomes more egalitarian. 
- Spatial organization - allowing participants to layout elements of the joint product in space increases mutual engagement by supporting fluid and improvised privacy and grouping.

The design question then becomes: How are these features used to inform design, especially in audio-only interfaces. Interestingly, in recent studies we found that implementing all the design features could actually reduce mutual engagement, possibly due to cognitive overload.

\subsection{Evaluation Techniques}

Through our studies we have iteratively refined a set of measures of mutual engagement based on analysis of patterns of participants' interaction, and a robust Mutual Engagement Questionnaire (MEQ) which can be used to compare different interfaces. These measures and questionnaires are suitably generic to be usable across different social music interfaces. Our measures of mutual engagement include:

- Number of contributions, edits, and deletions - excessive numbers of contributions in the music domain indicates low levels of mutual engagement.

- Amount of co-editing (i.e. editing each others' contributions) - increased constructive co-editing indicates increased mutual engagement.

- Spatial colocation - working together in the same part of a virtual space indicates mutual engagement.

- Evidence of convergence of musical ideas (i.e. alignment and repetition of musical motifs) indicates mutual engagement.

Measures of musical convergence between participants are problematic. We are currently investigating techniques to reliably identify convergence of musical ideas in social music making including using Music Information Retrieval techniques such as edit-distance and sub-sequence sampling. We believe that although these techniques focus on monophonic sources [11], they could have significant utility in understanding social music interaction in general.

In contrast, our Mutual Engagement Questionnaire (MEQ) is used to compare two or more user interfaces. In this approach participants use a number of user interfaces and then complete the MEQ of twelve questions from four categories (not conveyed to participants): Satisfaction with the product, Feelings of enjoyment or flow cf. [7], Sense of collaboration, and Usability. The comparative nature of the MEQ forces participants into making explicit distinctions between interfaces. Our MEQ would be suitable for comparing different social music interfaces and experiences, and would provide a good indicator of participants' preferences.

\section{Explorations of Multi-Person Musical Experiences}

We have been exploring mutual engagement in social music through a series of studies from interactive art through to group music composition. The main vehicle for this work has been a series of studies of distributed music making applications Daisyphone [3] and Daisyfield (forthcoming). We follow discussion of these systems with a brief description of other social music systems we have been exploring. 


\subsection{Daisyphone and Daisyfield}

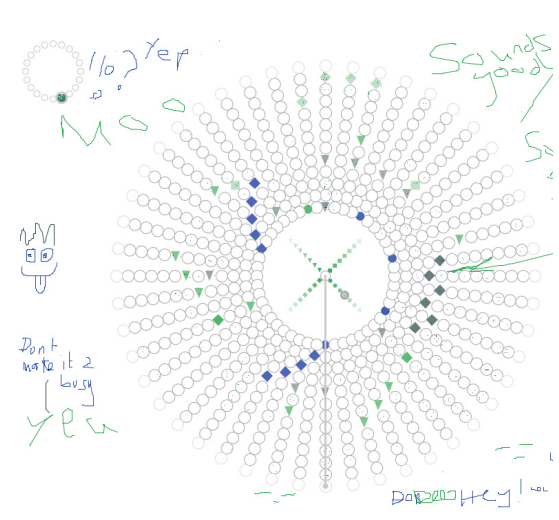

Figure 1. Daisyphone in use

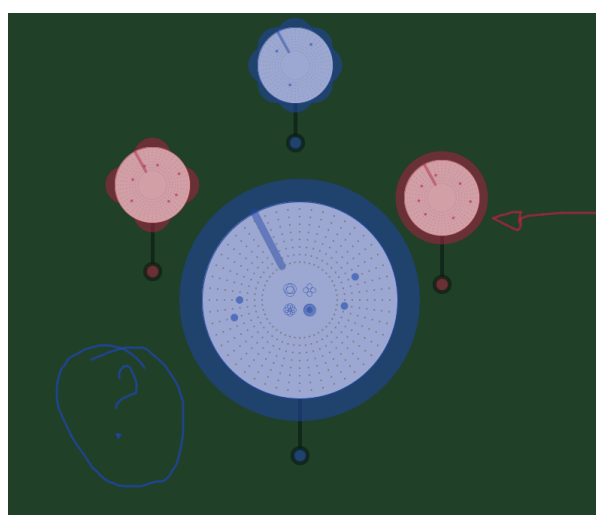

Figure 2. Daisyfield in use

Daisyphone (figure 1) and Daisyfield (figure 2) share a common underlying distributed, client-server architecture, which allows multiple participants to co-create short loops of music (1 minute) without being in the same physical space. At the heart of the user experience are loops which are shared between participants and can be coedited at will (there are no ownership controls). Loops are represented as Daisys with the notes of the loop laid out in a circular fashion. Daisyphone provides one shared loop, Daisyfield supports up to 12 shared loops, each represented as a separate Daisy arranged across the shared space. Indeed, Daisyfield is a development of Daisyphone which draws on our studies of naturalistic music improvisation [12] and composition [15]. In keeping with our design features, mutual awareness of action is supported by each participant having a unique colour in the interface, annotation is supported through free graphic drawing, and the whole interface is shared consistently between participants. Lowest notes are on the outsides of Daisys, and highest towards the centre (Persian scale of electro-acoustic sounds). The four shapes in the centre of each Daisy allow for selection of different sounds.

Using Daisyphone and Daisyfield we have explored the role of mutual awareness, persistence of musical contributions, graphical annotations, localization of sounds, and spatial arrangements. We have also used them to revise and validate our MEQ. Both systems have a set of features which make them particularly amenable to automated analysis of musical convergence: notes are played at a constant speed, and each note has the same duration. However, the interfaces allow several notes to be played at the same time which increases the complexity of applying pattern matching techniques. We shall be exploring these issues in ongoing research.

In studies we confirmed that providing shared annotation mechanisms and mutual awareness of identity of others significantly increased mutually engagement between participants as measured through our MEQ and correlated with our measures of mutual engagement [5]. This was manifested as more focused interaction between participants indicated by fewer contributions and edits of notes [ibid]. We also found that whilst the shared music making can happen without additional communication 
channels, when present, shared annotation mechanisms are used both for task management and social interaction, and there are more complimentary efforts in composition when shared annotations are provided [3][5]. Also, more annotations about quality of composition seems to indicate more mutual engagement.

Whilst Daisyphone and Daisyfield are unashamedly Graphical User Interfaces, we have also been exploring designing for mutual engagement in musical user interfaces which are not visually oriented. For instance, Stowell et al. [18] showed how rigorous HCI approaches could be used to evaluate people's engagement with and through musical interfaces - a novel beat-boxing synthesizer was evaluated using Discourse Analysis to explore engagement with the technology, and a live beat-tracking system was evaluated using a version of the classic Turing Test to evaluate participants' engagement with each other and the beat-tracking system. In contrast, we focused on the mutual awareness of actions design feature in designing the Serendiptichord [14] a wearable musical instrument whose design considers exploration of musical space, and the engagement of performer with instrument and audience. Similarly, mutual awareness of action and spatial organization were key factors in the design of $u P o i$ [17] - a guerilla multi-person interactive audiovisual experience intended to entice and engage participants with each other in unexpected and unusual situations which we observed in use at music festivals. Focusing on designing for mutual awareness of action and shared and consistent representations, Sensory Threads [4], is a multiperson mobile experience in which participants sense imperceptible phenomena around them through a responsive real-time soundscape. Our design features informed the design of the soundscape, ensuring that it conveyed the identity of participants clearly, and that there was clear auditory and spatial separation between sounds in the emergent virtual space. In all these non-visual designs we used our mutual engagement design features to drive the design to create a more engaging experience. From the feedback and observations we found that people did engage with each other, but we need to refine our evaluation techniques to work outside the laboratory. In contrast, our research on cross-modal collaborative work has explored the role of audio in workplace group interaction [13], focusing on task efficiency, but using the design features of mutual awareness to create effective collaboration environments. It would be interesting to explore how these systems could be further developed to support social music making through cross-modal interaction.

\section{Summary}

In this paper we presented our view on mutual engagement as the key to successful multi-person music making. We presented a set of design features and methods of evaluation which we feel could help inform the understanding of social behavior in music, and help to design more mutually engaging musical experiences. The work presented here is a small step in that direction. Future work will test our ideas on mutual engagement in different domains, and explore social music making using a range of modalities through cross-modal interaction.

Research supported by EPSRC grants EP/H042865/1, EP/E045235/1, GR/S81414/01. 


\section{Nick Bryan-Kinns}

\section{References}

[1] Ackerman, M. S., Starr, B., Hindus, D., and Mainwaring, S. D. Hanging on the 'wire: a field study of an audio-only media space. ACM Transactions on Computer-Human Interaction, 4, 1 (March 1997), 39-66. (1997).

[2] Barbosa, A. Displaced soundscapes: a survey of network systems for music and sonic art creation. Leonardo Music Journal, 13, 53-59. (2003).

[3] Bryan-Kinns, N. Daisyphone: The Design and Impact of a Novel Environment for Remote Group Music Improvisation. In Proceedings of DIS 2004, Boston, USA, 135-144. (2004).

[4]Bryan-Kinns, N., Airantzis, D., Angus, A., Fencott, R., Lane, G,, Lesage, F., Marshall, J., Martin, K., Roussos, G., Taylor, J., Warren, L., and Woods, O. Sensory Threads: Perceiving the Imperceptible. In Proceedings of 5th International Conference on Intelligent Environments (IE'09). (2009).

[5]Bryan-Kinns, N., and Hamilton, F. Identifying Mutual Engagement. Behaviour \& Information Technology. DOI: 10.1080/01449290903377103. (2009).

[6] Clark, H.H., and Brennan, S.E. Grounding in communication. In: L.B. Resnick, J. Levine, and S.D. Behrend, eds. Perspectives on socially shared cognition. Washington, DC: American Psychological Association, 127-149. (1991).

[7] Csikszentmihalyi, M. Flow: the psychology of optimal experience. New York: Harper Collins. (1991)

[8] Dobrian, C., and Koppelman, D. The 'E' in NIME: musical expression with new computer interfaces. In Proceedings of new interfaces for musical expression (NIME). Paris, France: IRCAM, Centre Pompidou, 277-282. (2006).

[9] Graff, G. Bob Geldof Pleads For Rock's Future At SXSW Keynote, The Hollywood Reporter, 17 March 2011. (2011).

[10] Gutwin, C., and Greenberg, S. A descriptive framework of workspace awareness for realtime groupware. Computer Supported Cooperative Work, 11, 411-446. (2002).

[11] Hanna, P., Robine, M., Ferraro, P., and Allali, J. Improvements of Alignment Algorithms for Polyphonic Music Retrieval. Computer Music Modeling and Retrieval 2008, Denmark (2008).

[12] Healey, P. G. T., Leach, J., and Bryan-Kinns, N. Inter-Play: Understanding Group Music Improvisation as a Form of Everyday Interaction. In Proceedings of Less is More - Simple Computing in an Age of Complexity, Microsoft Research Cambridge. (2005).

[13] Metatla, O., Bryan-Kinns, N., and Stockman, T. Constructing Relational Diagrams in Audio: The Multiple Perspective Hierarchical Approach. In Proceedings of ASSETS2008, Halifax, Canada. (2008).

[14] Murray-Browne, T., Mainstone, D., Bryan-Kinns, N., and Plumbley, M. D. The Serendiptichord: A Wearable Instrument for Conteporary Dance Performance. To appear in Proceedings of the 128th Convention of the Audio Engineering Society, London, UK. (2010).

[15] Nabavian, S., and Bryan-Kinns, N. Analysing Group Creativity: A Distributed Cognitive Study of Joint Music Composition. In Proceedings of Cognitive Science, 1856-1861. (2006).

[16] Sawyer, K. R. Group Creativity: Music, Theater, Collaboration. New Jersey: LEA. (2003).

[17] Sheridan, J. G., and Bryan-Kinns, N. Designing for Performative Tangible Interaction. International Journal of Arts and Technology. Special Issue on Tangible and Embedded Interaction, 1, 3-4, 288-308. (2008).

[18] Stowell, D., Robertson, A., Bryan-Kinns, N., and Plumbley, M. D. Evaluation of live human-computer music-making: quantitative and qualitative approaches. International Journal of Human-Computer Studies, 67, 960-975. (2009).

[19] Wanderley, M. M., and Orio, N. Evaluation of input devices for musical expression: Borrowing tools from HCI. Computer Music Journal, Vol. 26, No. 3, 62-76. (2002). 